\title{
VD-BERT: A Unified Vision and Dialog Transformer with BERT
}

\author{
Yue Wang ${ }^{1 *}$, Shafiq Joty ${ }^{2}$, Michael R. Lyu ${ }^{1}$, Irwin King ${ }^{1}$, Caiming Xiong ${ }^{2}$, and Steven C.H. Hoi ${ }^{2}$ \\ ${ }^{1}$ Department of Computer Science and Engineering \\ The Chinese University of Hong Kong, HKSAR, China \\ ${ }^{2}$ Salesforce Research \\ ${ }^{1}$ \{yuewang, lyu, king\}@cse.cuhk.edu.hk \\ 2 \{sjoty, cxiong, shoi\}esalesforce.com
}

\begin{abstract}
Visual dialog is a challenging vision-language task, where a dialog agent needs to answer a series of questions through reasoning on the image content and dialog history. Prior work has mostly focused on various attention mechanisms to model such intricate interactions. By contrast, in this work, we propose VD-BERT, a simple yet effective framework of unified vision-dialog Transformer that leverages the pretrained BERT language models for Visual Dialog tasks. The model is unified in that (1) it captures all the interactions between the image and the multi-turn dialog using a single-stream Transformer encoder, and (2) it supports both answer ranking and answer generation seamlessly through the same architecture. More crucially, we adapt BERT for the effective fusion of vision and dialog contents via visually grounded training. Without the need of pretraining on external vision-language data, our model yields new state of the art, achieving the top position in both single-model and ensemble settings (74.54 and 75.35 NDCG scores) on the visual dialog leaderboard. Our code and pretrained models are released at https: //github.com/salesforce/VD-BERT.
\end{abstract}

\section{Introduction}

Visual Dialog (or VisDial) aims to build an AI agent that can answer a human's questions about visual content in a natural conversational setting (Das et al., 2017). Unlike the traditional single-turn Visual Question Answering (VQA) (Antol et al., 2015), the agent in VisDial requires to answer questions through multiple rounds of interactions together with visual content understanding.

The primary research direction in VisDial has been mostly focusing on developing various attention mechanisms (Bahdanau et al., 2015) for a bet-

\footnotetext{
*This work was mainly done when Yue Wang was an intern at Salesforce Research Asia, Singapore.
}

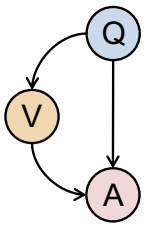

(a) Most VQA

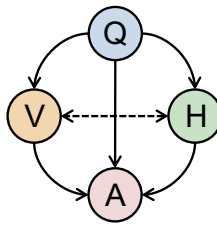

(b) Most VisDial

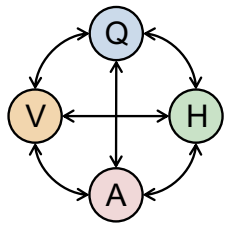

(c) Our VD-BERT
Figure 1: Attention flow direction illustration. V: vision, H: dialog history, Q: question, A: answer. The arrow denotes the attention flow direction and the dashed line represents an optional connection.

ter fusion of vision and dialog contents. Compared to VQA that predicts an answer based only on the question about the image (Figure 1(a)), VisDial needs to additionally consider the dialog history. Typically, most of previous work (Niu et al., 2019; Gan et al., 2019; Kang et al., 2019) uses the question as a query to attend to relevant image regions and dialog history, where their interactions are usually exploited to obtain better visual-historical cues for predicting the answer. In other words, the attention flow in these methods is unidirectional - from question to the other components (Figure 1(b)).

By contrast, in this work, we allow for bidirectional attention flow between all the entities using a unified Transformer (Vaswani et al., 2017) encoder, as shown in Figure 1(c). In this way, all the entities simultaneously play the role of an "information seeker" (query) and an "information provider" (key-value), thereby fully unleashing the potential of attention similar to Schwartz et al. (2019). We employ the Transformer as the encoding backbone due to its powerful representation learning capability exhibited in pretrained language models like BERT (Devlin et al., 2019). Inspired by its recent success in vision-language pretraining, we further extend BERT to achieve simple yet effective fusion of vision and dialog contents in VisDial tasks.

Recently several emerging works have attempted to adapt BERT for multimodal tasks (Sun et al., 
2019; Lu et al., 2019; Tan and Bansal, 2019; Zhou et al., 2020). They often use self-supervised objectives to pretrain BERT-like models on large-scale external vision-language data and then fine-tune on downstream tasks. This has led to compelling results in tasks such as VQA, image captioning, image retrieval (Young et al., 2014), and visual reasoning (Suhr et al., 2019). However, it is still unclear how visual dialog may benefit from such vision-language pretraining due to its unique multiturn conversational structure. Specifically, each image in the VisDial dataset is associated with up to 10 dialog turns, which contain much longer contexts than either VQA or image captioning.

In this paper, we present VD-BERT, a novel unified vision-dialog Transformer framework for VisDial tasks. Specifically, we first encode the image into a series of detected objects and feed them into a Transformer encoder together with the image caption and multi-turn dialog. We initialize the encoder with BERT for better leveraging the pretrained language representations. To effectively fuse features from the two modalities, we make use of two visually grounded training objectives Masked Language Modeling (MLM) and Next Sentence Prediction (NSP). Different from the original MLM and NSP in BERT, we additionally take the visual information into account when predicting the masked tokens or the next answer.

VisDial models have been trained in one of two settings: discriminative or generative. In the discriminative setting, the model ranks a pool of answer candidates, whereas the generative setting additionally allows the model to generate the answers. Instead of employing two types of decoders like prior work, we rely on a unified Transformer architecture with two different self-attention masks (Dong et al., 2019) to seamlessly support both settings. During inference, our VD-BERT either ranks the answer candidates according to their NSP scores or generates the answer sequence by recursively applying the MLM operations. We further fine-tune our model on dense annotations that specify the relevance score for each answer candidate with a ranking optimization module.

In summary, we make the following contributions:

- To the best of our knowledge, our work serves as one of the first attempts to explore pretrained language models for visual dialog. We showcase that BERT can be effectively adapted to this task with simple visually grounded training for capturing the intricate vision-dialog interactions. Besides, our VD-BERT is the first unified model that supports both discriminative and generative training settings without explicit decoders.

- We conduct extensive experiments not only to analyze how our model performs with various training aspects $(\S 5.2)$ and fine-tuning on dense annotations (§5.3), but also to interpret it via attention visualization ( $\$ 5.4)$, shedding light on future transfer learning research for VisDial tasks.

- Without the need to pretrain on external visionlanguage data, our model yields new state-of-theart results in discriminative setting and promising results in generative setting on the visual dialog benchmarks ( $\$ 5.1)$.

\section{Related Work}

Visual Dialog. The Visual Dialog task has been recently proposed by Das et al. (2017), where a dialog agent needs to answer a series of questions grounded by an image. It is one of the most challenging vision-language tasks that require not only to understand the image content according to texts, but also to reason through the dialog history. Previous work (Lu et al., 2017; Seo et al., 2017; Wu et al., 2018; Kottur et al., 2018; Jiang et al., 2020; Yang et al., 2019; Guo et al., 2019a; Niu et al., 2019) focuses on developing a variety of attention mechanisms to model the interactions among entities including image, question, and dialog history. For example, Kang et al. (2019) proposed DAN, a dual attention module to first refer to relevant contexts in the dialog history, and then find indicative image regions. ReDAN, proposed by Gan et al. (2019), further explores the interactions between image and dialog history via multi-step reasoning.

Different from them, we rely on the selfattention mechanism within a single-stream Transformer encoder to capture such interactions in a unified manner and derive a "holistic" contextualized representation for all the entities. Similar to this, Schwartz et al. (2019) proposed FGA, a general factor graph attention that can model interactions between any two entities but in a pairwise manner. There are recent works (Nguyen et al., 2019; Agarwal et al., 2020) also applying the Transformer to model the interactions among many entities. However, their models neglect the important early interaction of the answer entity and cannot naturally leverage the pretrained language representations from BERT like ours. 


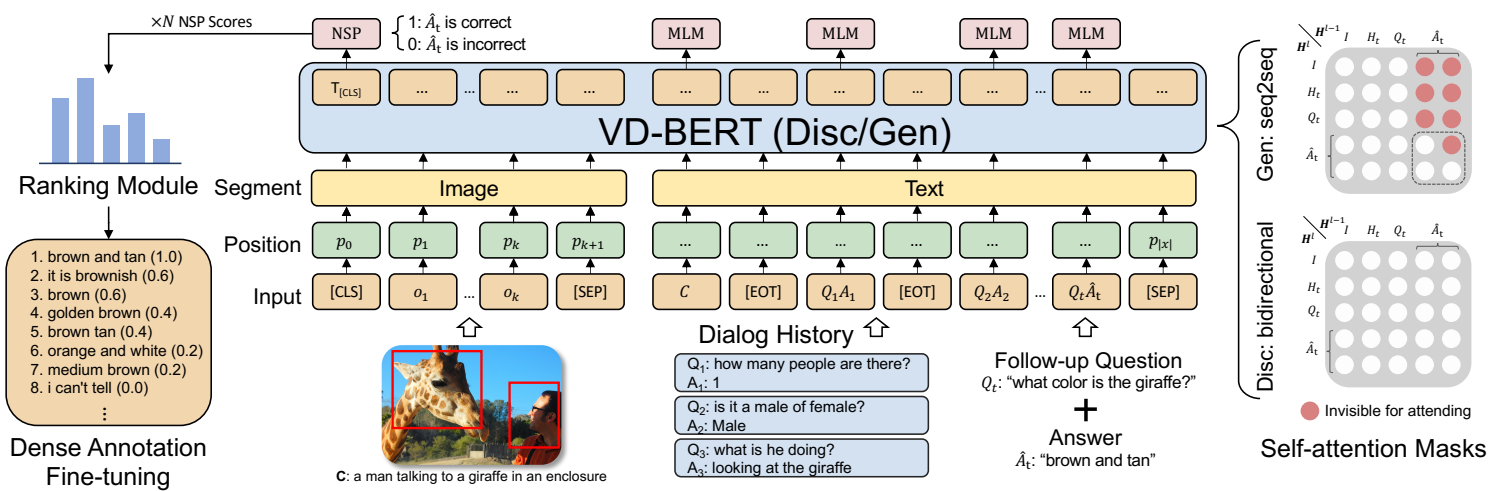

Figure 2: The model architecture of our unified VD-BERT for both discriminative and generative settings.

Regarding the architecture, our model mainly differs from previous work in two facets: first, unlike most prior work that considers answer candidates only at the final similarity computation layer, our VD-BERT integrates each answer candidate at the input layer to enable its early and deep fusion with other entities, similar to Schwartz et al. (2019); second, existing models adopt an encoderdecoder framework (Sutskever et al., 2014) with two types of decoder for the discriminative and generative settings separately, while we instead adopt a unified Transformer encoder with two different self-attention masks (Dong et al., 2019) to seamlessly support both settings without extra decoders.

Pretraining in Vision and Language. Pretrained language models like BERT (Devlin et al., 2019) have boosted performance greatly in a broad set of NLP tasks. In order to benefit from the pretraining, there are many recent works on extending BERT for vision and language pretraining. They typically employ the Transformer encoder as the backbone with either a two-stream architecture to encode text and image independently such as ViLBERT (Lu et al., 2019) and LXMERT (Tan and Bansal, 2019), or a single-stream architecture to encode both text and image together, such as B2T2 (Alberti et al., 2019), Unicoder-VL (Li et al., 2020), VisualBERT (Li et al., 2019), VL-BERT (Su et al., 2020), and UNITER (Chen et al., 2019). Our VD-BERT belongs to the second group. These models yield prominent improvements mainly on vision-language understanding tasks like VQA, image retrieval (Young et al., 2014), and visual reasoning (Suhr et al., 2019; Zellers et al., 2019).

More recently, Zhou et al. (2020) proposed VLP which also allows generation using a unified Transformer with various self-attention masks (Dong et al., 2019). Their model was proposed for VQA and image captioning. Our model is inspired by
VLP and specifically tailored for the visual dialog task. Most closely related to this paper is the concurrent work VisDial-BERT by Murahari et al. (2019), who also employ pretrained models (i.e., ViLBERT) for visual dialog. Our work has two major advantages over VisDial-BERT: first, VDBERT supports both discriminative and generative settings while theirs is restricted to only the discriminative setting; second, we do not require to pretrain on large-scale external vision-language datasets like theirs and still yield better performance ( $(5.1)$.

\section{The VD-BERT Model}

We first formally describe the visual dialog task. Given a question $Q_{t}$ grounded on an image $I$ at $t$-th turn, as well as its dialog history formulated as $H_{t}=\left\{C,\left(Q_{1}, A_{1}\right), \ldots,\left(Q_{t-1}, A_{t-1}\right)\right\}$ (where $C$ denotes the image caption), the agent is asked to predict its answer $A_{t}$ by ranking a list of 100 answer candidates $\left\{\hat{A}_{t}^{1}, \hat{A}_{t}^{2}, \ldots, \hat{A}_{t}^{100}\right\}$. In general, there are two types of decoder to predict the answer: a discriminative decoder that ranks the answer candidates and is trained with a cross entropy loss, or a generative decoder that synthesizes an answer and is trained with a maximum log-likelihood loss.

Figure 2 shows the overview of our approach. First, we employ a unified vision-dialog Transformer to encode both the image and dialog history, where we append an answer candidate $\hat{A}_{t}$ in the input to model their interactions in an early fusion manner (§3.1). Next, we adopt visually grounded MLM and NSP objectives to train the model for effective vision and dialog fusion using two types of self-attention masks - bidirectional and seq2seq. This allows our unified model to work in both discriminative and generative settings (§3.2). Lastly, we devise a ranking optimization module to further fine-tune on the dense annotations ( $\$ 3.3)$. 


\subsection{Vision-Dialog Transformer Encoder}

Vision Features. Following previous work, we employ Faster R-CNN (Ren et al., 2015) pretrained on Visual Genome (Krishna et al., 2017) to extract the object-level vision features. Let $O_{I}=\left\{o_{1}, \ldots, o_{k}\right\}$ denote the vision features for an image $I$, where each object feature $o_{i}$ is a 2048-d Region-of-Interest (RoI) feature and $k$ is the number of the detected objects (fixed to 36 in our setting). As there is no natural orders among these objects, we adopt normalized bounding box coordinates as the spatial location. Specifically, let $\left(x_{1}, y_{1}\right)$ and $\left(x_{2}, y_{2}\right)$ be the coordinates of the bottom-left and top-right corner of the $i$-th object, its location information is encoded into a 5 -d vector: $p_{i}=\left(\frac{x_{1}}{W}, \frac{y_{1}}{H}, \frac{x_{2}}{W}, \frac{y_{2}}{H}, \frac{\left(x_{2}-x_{1}\right)\left(y_{2}-y_{1}\right)}{W H}\right)$, where $W$ and $H$ respectively denote the width and height of the input image, and the last element is the relative area of the object. We extend $p_{i}$ with its class id and confidence score for a richer representation.

Language Features. We pack all the textual elements (caption and multi-turn dialog) into a long sequence. We employ WordPiece tokenizer (Wu et al., 2016) to split it into a word sequence $\mathbf{w}$, where each word is embedded with an absolute positional code following Devlin et al. (2019).

Cross-Modality Encoding. To feed both image and text into the Transformer encoder, we integrate the image objects with language elements into a whole input sequence. Similar to BERT, we use special tokens like [CLS] to denote the beginning of the sequence, and [SEP] to separate the two modalities. Moreover, to inject the multi-turn dialog structure into the model, we utilize a special token [EOT] to denote end of turn (Whang et al., 2019), which informs the model when the dialog turn ends. As such, we prepare the input sequence into the format as $\mathbf{x}=\left([\mathrm{CLS}], o_{1}, \ldots, o_{k},[\mathrm{SEP}]\right.$, $C$, [EОT], $Q_{1} A_{1}$, [EОT] $, \ldots, Q_{t} \hat{A}_{t}$, [SEP ] ). To notify the model for the answer prediction, we further insert a [PRED] token between the $Q_{t} \hat{A}_{t}$ pair. Finally, each input token embedding is combined with its position embedding and segment embedding ( 0 or 1 , indicating whether it is image or text) with layer normalization (Ba et al., 2016).

Transformer Backbone. We denote the embedded vision-language inputs as $\mathbf{H}^{0}=\left[\mathbf{e}_{1}, \ldots, \mathbf{e}_{|\mathbf{x}|}\right]$ and then encode them into multiple levels of contextual representations $\mathbf{H}^{l}=\left[\mathbf{h}_{1}^{l}, \ldots, \mathbf{h}_{\mid \mathbf{x}}^{l}\right]$ using $L$-stacked Transformer blocks, where the $l$-th Transformer block is denoted as $\mathbf{H}^{l}=$ Transformer $\left(\mathbf{H}^{l-1}\right), l \in[1, L]$. Inside each Transformer block, the previous layer's output $\mathbf{H}^{l-1} \in$ $\mathbb{R}^{|\mathbf{x}| \times d_{h}}$ is aggregated using the multi-head selfattention (Vaswani et al., 2017):

$$
\begin{gathered}
\mathbf{Q}=\mathbf{H}^{l-1} \mathbf{W}_{l}^{Q}, \mathbf{K}=\mathbf{H}^{l-1} \mathbf{W}_{l}^{K}, \mathbf{V}=\mathbf{H}^{l-1} \mathbf{W}_{l}^{V}, \\
\mathbf{M}_{i j}= \begin{cases}0, & \text { allow to attend, } \\
-\infty, & \text { prevent from attending, }\end{cases} \\
\mathbf{A}_{l}=\operatorname{softmax}\left(\frac{\mathbf{Q K}}{\sqrt{d_{k}}}+\mathbf{M}\right) \mathbf{V},
\end{gathered}
$$

where $\mathbf{W}_{l}^{Q}, \mathbf{W}_{l}^{K}, \mathbf{W}_{l}^{V} \in \mathbb{R}^{d_{h} \times d_{k}}$ are learnable weights for computing the queries, keys, and values respectively, and $\mathbf{M} \in \mathbb{R}^{|\mathbf{x}| \times|\mathbf{x}|}$ is the self-attention mask that determines whether tokens from two layers can attend each other. Then $\mathbf{A}_{l}$ is passed into a feedforward layer to compute $\mathbf{H}^{l}$ for the next layer.

\subsection{Visually Grounded Training Objectives}

We use two visually grounded training objectivesmasked language modeling (MLM) and next sentence prediction (NSP) to train our VD-BERT. Particularly, we aim to capture dense interactions among both inter-modality (i.e., image-dialog) and intra-modality (i.e., image-image, dialog-dialog).

Similar to MLM in BERT, $15 \%$ tokens in the text segment (including special tokens like [EOT] and $[\mathrm{SEP}]$ ) are randomly masked out and replaced with a special token [MASK]. The model is then required to recover them based not only on the surrounding tokens $\mathbf{w}_{\backslash m}$ but also on the image $I$ :

$$
\mathcal{L}_{M L M}=-E_{(I, \mathbf{w}) \sim D} \log P\left(w_{m} \mid \mathbf{w}_{\backslash m}, I\right),
$$

where $w_{m}$ refers to the masked token and $D$ denotes the training set. Following Zhou et al. (2020), we do not conduct similar masked object/region modeling in the image segment.

As for NSP, instead of modeling the relationship between two sentences (as in BERT) or the matching of an image-text pair (as in other visionlanguage pretraining models like ViLBERT), VDBERT aims to predict whether the appended answer candidate $\hat{A}_{t}$ is correct or not based on the joint understanding of the image and dialog history:

$$
\mathcal{L}_{N S P}=-E_{(I, \mathbf{w}) \sim D} \log P(y \mid S(I, \mathbf{w})),
$$

where $y \in\{0,1\}$ indicates whether $\hat{A}_{t}$ is correct, and $S(\cdot)$ is a binary classifier to predict the probability based on the [CLS] representation $\mathrm{T}_{\text {[CLS] }}$ at the final layer. Below we introduce the discriminative and generative settings of VD-BERT. 
Discriminative Setting. For training in the discriminative setting, we transform the task of selecting an answer into a point-wise binary classification problem. Specifically, we sample an answer $\hat{A}_{t}$ from the candidate pool and append it to the input sequence, and ask the NSP head to distinguish whether the sampled answer is correct or not. We employ the bidirectional self-attention mask to allow all the tokens to attend to each other by setting the mask matrix $\mathbf{M}$ in Eq. (2) to all 0s. To avoid imbalanced class distribution, we keep the ratio of positive and negative instances to $1: 1$ in each epoch. To encourage the model to penalize more on negative instances, we randomly resample a negative example from the pool of 99 negatives w.r.t. every positive one at different epochs. During inference, we rank the answer candidates according to the positive class score of their NSP heads.

Generative Setting. In order to autoregressively generate an answer, we also train VD-BERT with the sequence-to-sequence (seq2seq) self-attention mask (Dong et al., 2019). For this, we divide the input sequence to each Transformer block into two subsequences, context and answer:

$$
\mathbf{x} \triangleq(I, \mathbf{w})=(\underbrace{I, H_{t}, Q_{t}}_{\text {context }}, \hat{A}_{t}) .
$$

We allow tokens in the context to be fully visible for attending by setting the left part of $\mathbf{M}$ to all $0 \mathrm{~s}$. For the answer sequence, we mask out (by setting $-\infty$ in $\mathbf{M}$ ) the "future" tokens to get autoregressive attentions (see the red dots in Figure 2).

During inference, we rely on the same unified Transformer encoder with sequential MLM operations without an explicit decoder. Specifically, we recursively append a [MASK] token to the end of the sequence to trigger a one-step prediction and then replace it with the predicted token for the next token prediction. The decoding process is based on greedy sampling and terminated when a [SEP] is emitted, and the resulting log-likelihood scores will be used for ranking the answer candidates.

\subsection{Fine-tuning with Rank Optimization}

As some answer candidates may be semantically similar (e.g., "brown and tan" vs "brown" in Figure 2), VisDial v1.0 additionally provides dense annotations that specify real-valued relevance scores for the 100 answer candidates, $\left[s_{1}, \ldots, s_{100}\right]$ with $s_{i} \in[0,1]$. To fine-tune on this, we combine the
NSP scores from the model for all answer candidates together into a vector $\left[p_{1}, \ldots, p_{100}\right]$.

As dense annotation fine-tuning is typically a Learning to Rank (LTR) problem, we can make use of some ranking optimization methods (see the Appendix B.1 for more details). We adopt ListNet (Cao et al., 2007) with the top-1 approximation as the ranking module for VD-BERT:

$$
\begin{gathered}
\mathcal{L}_{\text {List Net }}=-\sum_{i=1}^{N} f\left(s_{i}\right) \log \left(f\left(p_{i}\right)\right), \\
f\left(x_{i}\right)=\frac{\exp \left(x_{i}\right)}{\sum_{j=1}^{N} \exp \left(x_{j}\right)}, i=1, \ldots, N .
\end{gathered}
$$

Here $N$ is the number of answer candidates. For training efficiency, we sub-sample the candidate list and use only $N=30$ answers (out of 100) for each instance. To better leverage the contrastive signals from the dense annotations, the sub-sampling method first picks randomly the candidates with non-zero relevance scores, and then it picks the ones from zero scores (about $12 \%$ of candidates are non-zero on average).

\section{Experimental Setup}

Datasets. We evaluate our model on the VisDial v0.9 and v1.0 datasets (Das et al., 2017). Specifically, v0.9 contains a training set of 82,783 images and a validation set of 40, 504 images. The v1.0 dataset combines the training and validation sets of v0.9 into one training set and adds another 2,064 images for validation and 8,000 images for testing (hosted blindly in the task organizers' server). Each image is associated with one caption and 10 question-answer pairs. For each question, it is paired with a list of 100 answer candidates, one of which is regarded as the correct answer.

For the v1.0 validation split and a part of v1.0 train split (2,000 images), extra dense annotations for the answer candidates are provided to make the evaluation more reasonable. The dense annotation specifies a relevance score for each answer candidate based on the fact that some candidates with similar semantics to the ground truth answer can also be considered as correct or partially correct, e.g., "brown and tan" and "brown" in Figure 2.

Evaluation Metric. Following Das et al. (2017), we evaluate our model using the ranking metrics like Recall@K $(\mathrm{K} \in\{1,5,10\})$, Mean Reciprocal Rank (MRR), and Mean Rank, where only one 
answer is considered as correct. Since the 2018 VisDial challenge (after the acquisition of dense annotations), NDCG metric that considers the relevance degree of each answer candidate, has been adopted as the main metric to determine the winner.

Configurations. We use BERT $\mathrm{BASE}_{\mathrm{BS}}$ as the backbone, which consists of 12 Transformer blocks, each with 12 attention heads and a hidden state dimensions of 768 . We keep the max input sequence length (including 36 visual objects) to 250 . We use Adam (Kingma and Ba, 2015) with an initial learning rate of $3 e-5$ and a batch size of 32 to train our model. A linear learning rate decay schedule with a warmup of 0.1 is employed. We first train VD-BERT for 30 epochs on a cluster of 4 V100 GPUs with 16G memory using MLM and NSP losses (with equal coefficients). Here we only utilize one previous dialog turn for training efficiency. For instances where the appended answer candidate is incorrect, we do not conduct MLM on the answer sequence to reduce the noise introduced by the negative samples. After that, we train for another 10 epochs with full dialog history using either NSP in the discriminative setting or MLM on the answer sequence in the generative setting. For dense annotation fine-tuning in the discriminative setting, we train with the ListNet loss for 5 epochs.

\section{Results and Analysis}

We first compare VD-BERT with state-of-the-art models on VisDial datasets ( $\$ 5.1)$. Then we conduct ablation studies to examine various aspects of our model ( $\$ 5.2)$, followed by an in-depth analysis of fine-tuning on dense annotations ( $§ 5.3)$. Lastly, we interpret how it attains the effective fusion of vision and dialog via attention visualization (§5.4).

\subsection{Main Results}

Comparison. We consider state-of-the-art published baselines, including NMN (Hu et al., 2017), CorefNMN (Kottur et al., 2018), GNN (Zheng et al., 2019), FGA (Schwartz et al., 2019), DVAN (Guo et al., 2019b), RvA (Niu et al., 2019), DualVD (Jiang et al., 2020), HACAN (Yang et al., 2019), Synergistic (Guo et al., 2019a), DAN (Kang et al., 2019), ReDAN (Gan et al., 2019), CAG (Guo et al., 2020), Square (Kim et al., 2020), MCA (Agarwal et al., 2020), MReal-BDAI and P1_P2 (Qi et al., 2020). We further report re-

\begin{tabular}{|c|c|c|c|c|c|c|c|}
\hline & Model & $\mathrm{NDCG} \uparrow$ & $\mathrm{MRR} \uparrow$ & $\mathrm{R} @ 1 \uparrow$ & $\mathrm{R} @ 5 \uparrow$ & $\mathrm{R} @ 10 \uparrow$ & Mean $\downarrow$ \\
\hline \multirow{18}{*}{ 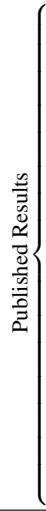 } & NMN & 58.10 & 58.80 & 44.15 & 76.88 & 86.88 & 4.81 \\
\hline & CorefNMN & 54.70 & 61.50 & 47.55 & 78.10 & 88.80 & 4.40 \\
\hline & GNN & 52.82 & 61.37 & 47.33 & 77.98 & 87.83 & 4.57 \\
\hline & FGA & 52.10 & 63.70 & 49.58 & 80.97 & 88.55 & 4.51 \\
\hline & DVAN & 54.70 & 62.58 & 48.90 & 79.35 & 89.03 & 4.36 \\
\hline & RvA & 55.59 & 63.03 & 49.03 & 80.40 & 89.83 & 4.18 \\
\hline & DualVD & 56.32 & 63.23 & 49.25 & 80.23 & 89.70 & 4.11 \\
\hline & HACAN & 57.17 & 64.22 & 50.88 & 80.63 & 89.45 & 4.20 \\
\hline & Synergistic & 57.32 & 62.20 & 47.90 & 80.43 & 89.95 & 4.17 \\
\hline & Synergistic $^{\dagger}$ & 57.88 & 63.42 & 49.30 & 80.77 & $\underline{90.68}$ & 3.97 \\
\hline & DAN & 57.59 & 63.20 & 49.63 & 79.75 & 89.35 & 4.30 \\
\hline & DAN $^{\dagger}$ & 59.36 & 64.92 & 51.28 & $\underline{81.60}$ & 90.88 & 3.92 \\
\hline & $\operatorname{ReDAN}^{\dagger}$ & 64.47 & 53.73 & 42.45 & 64.68 & 75.68 & 6.64 \\
\hline & CAG & 56.64 & 63.49 & 49.85 & 80.63 & 90.15 & 4.11 \\
\hline & Square $^{\dagger}$ & 60.16 & 61.26 & 47.15 & 78.73 & 88.48 & 4.46 \\
\hline & $\mathrm{MCA}^{*}$ & 72.47 & 37.68 & 20.67 & 56.67 & 72.12 & 8.89 \\
\hline & MReal-BDAI $^{* *}$ & 74.02 & 52.62 & 40.03 & 68.85 & 79.15 & 6.76 \\
\hline & P1_P2 ${ }^{\dagger *}$ & $\underline{74.91}$ & 49.13 & 36.68 & 62.98 & 78.55 & 7.03 \\
\hline \multirow{11}{*}{ 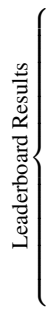 } & $\mathrm{LF}$ & 45.31 & 55.42 & 40.95 & 72.45 & 82.83 & 5.95 \\
\hline & HRE & 45.46 & 54.16 & 39.93 & 70.45 & 81.50 & 6.41 \\
\hline & MN & 47.50 & 55.49 & 40.98 & 72.30 & 83.30 & 5.92 \\
\hline & MN-Att & 49.58 & 56.90 & 42.42 & 74.00 & 84.35 & 5.59 \\
\hline & LF-Att & 49.76 & 57.07 & 42.08 & 74.82 & 85.05 & 5.41 \\
\hline & MS ConvAI & 55.35 & 63.27 & 49.53 & 80.40 & 89.60 & 4.15 \\
\hline & UET-VNU $^{\dagger}$ & 57.40 & 59.50 & 45.50 & 76.33 & 85.82 & 5.34 \\
\hline & MVAN & 59.37 & 64.84 & $\underline{51.45}$ & 81.12 & 90.65 & 3.97 \\
\hline & SGLNs $^{\dagger}$ & 61.27 & 59.97 & $\overline{45.68}$ & 77.12 & 87.10 & 4.85 \\
\hline & VisDial-BERT* & 74.47 & 50.74 & 37.95 & 64.13 & 80.00 & 6.28 \\
\hline & Tohoku-CV ${ }^{\dagger *}$ & 74.88 & 52.14 & 38.93 & 66.60 & 80.65 & 6.53 \\
\hline \multirow{3}{*}{ 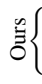 } & VD-BERT & 59.96 & 65.44 & 51.63 & 82.23 & $\underline{90.68}$ & 3.90 \\
\hline & VD-BERT* & 74.54 & 46.72 & 33.15 & 61.58 & $\overline{77.15}$ & 7.18 \\
\hline & VD-BERT $^{\dagger *}$ & 75.35 & 51.17 & 38.90 & 62.82 & 77.98 & 6.69 \\
\hline
\end{tabular}

Table 1: Summary of results on the test-std split of VisDial v1.0 dataset. The results are reported by the test server. " $\nmid$ " denotes ensemble model and " $*$ " indicates fine-tuning on dense annotations. The "^" denotes higher value for better performance and " $\downarrow$ " is the opposite. The best and second-best results in each column are in bold and underlined respectively.

sults from the leaderboard ${ }^{1}$ for a more up-to-date comparison, where some can be found in the arXiv, such as MVAN (Park et al., 2020), SGLNs (Kang et al., 2020), VisDial-BERT (Murahari et al., 2019), and Tohoku-CV (Nguyen et al., 2019).

Results on VisDial v1.0 test-std. We report the comparison results on VisDial v1.0 test-std split in Table 1 and make the following observations.

- New state of the art for both single-model and ensemble settings. Our single-model VD-BERT significantly outperforms all of its single-model counterparts across various metrics, even including some ensemble variants such as Synergistic, DAN (except R@ 10), and ReDAN (except NDCG). With further fine-tuning on dense annotations, the NDCG score increases quite sharply, from 59.96 to 74.54 with nearly $15 \%$ absolute improvement, setting a new state of the art in the single-model setting. This indicates that dense annotation finetuning plays a crucial role in boosting the NDCG

\footnotetext{
${ }^{1}$ https://evalai.cloudcv.org/web/ challenges/challenge-page/161/ leaderboard/483\# leaderboardrank-1
} 


\begin{tabular}{lccccc}
\hline \multirow{2}{*}{ Model } & MRR $\uparrow$ & $\mathrm{R} @ 1 \uparrow$ & $\mathrm{R} @ 5 \uparrow$ & $\mathrm{R} @ 10 \uparrow$ & Mean $\downarrow$ \\
\cline { 2 - 6 } & \multicolumn{5}{c}{ Discriminative/Generative } \\
\hline LF & $58.07 / 51.99$ & $43.82 / 41.83$ & $74.68 / 61.78$ & $84.07 / 67.59$ & $5.78 / 17.07$ \\
HRE & $58.46 / 52.37$ & $44.67 / 42.29$ & $74.50 / 62.18$ & $84.22 / 67.92$ & $5.72 / 17.07$ \\
HREA & $58.68 / 52.42$ & $44.82 / 42.28$ & $74.81 / 62.33$ & $84.36 / 68.17$ & $5.66 / 16.79$ \\
MN & $59.65 / 52.59$ & $45.55 / 42.29$ & $76.22 / 62.85$ & $85.37 / 68.88$ & $5.46 / 17.06$ \\
HCIAE & $62.22 / 54.67$ & $48.48 / 44.35$ & $78.75 / 65.28$ & $87.59 / 71.55$ & $4.81 / 14.23$ \\
CoAtt & $63.98 / 55.78$ & $50.29 / 46.10$ & $80.71 / 65.69$ & $88.81 / 71.74$ & $4.47 / 14.43$ \\
RvA & $66.34 / 55.43$ & $52.71 / 45.37$ & $\underline{82.97 / 65.27}$ & $\underline{90.73 / 72.97}$ & $\mathbf{3 . 9 3 / 1 0 . 7 1}$ \\
DVAN & $\underline{66.67 / 55.94}$ & $\underline{53.62 / 46.58}$ & $82.85 / 65.50$ & $90.72 / 71.25$ & $\mathbf{3 . 9 3 / 1 4 . 7 9}$ \\
\hline VD-BERT & $\mathbf{7 0 . 0 4 / 5 5 . 9 5}$ & $\mathbf{5 7 . 7 9 / 4 6 . 8 3}$ & $\mathbf{8 5 . 3 4 / 6 5 . 4 3}$ & $\mathbf{9 2 . 6 8 / 7 2 . 0 5}$ & $\underline{4.04 / 13.18}$ \\
\hline
\end{tabular}

Table 2: Discriminative and generative results of various models on the val split of VisDial v0.9 dataset.

scores. Moreover, our designed ensemble version yields new state of the art (75.35 NDCG), outperforming the 2019 VisDial challenge winner MRealBDAI (74.02 NDCG) by over 1.3 absolute points.

- Inconsistency between NDCG and other metrics. While dense annotation fine-tuning yields huge improvements on NDCG, we also notice that it has a severe countereffect on other metrics, e.g., reducing the MRR score from 65.44 to 46.72 for VD-BERT. Such a phenomenon has also been observed in other recent models, such as MReal-BDAI, VisDialBERT, Tohoku-CV Lab, and P1_P2, whose NDCG scores surpass others without dense annotation finetuning by at least around $10 \%$ absolute points while other metrics drop dramatically. We provide a detailed analysis of this phenomenon in $\S 5.3$.

- Our VD-BERT is simpler and more effective than VisDial-BERT. VisDial-BERT is a concurrent work to ours that also exploits vision-language pretrained models for visual dialog. It only reports the single-model performance of 74.47 NDCG. Compare to that, our VD-BERT achieves slightly better results (74.54 NDCG), however, note that we did not pretrain on large-scale external vision-language datasets like Conceptual Captions (Sharma et al., 2018) and VQA (Antol et al., 2015) as VisDialBERT does. Besides, while VisDial-BERT does not observe improvements by ensembling, we endeavor to design an effective ensemble strategy to increase the NDCG score to 75.35 for VD-BERT.

Results on VisDial v0.9 val. We further show both discriminative and generative results on v0.9 val split in Table 2. For comparison, we choose LF, HRE, HREA, MN (Das et al., 2017), HCIAE (Lu et al., 2017), CoAtt (Wu et al., 2018), RvA, and DVAN as they contain results in both settings on the v0.9 val split. These models employ dual decoders for each setting separately. Our model continues to yield much better results in the discriminative setting (e.g., 70.04 MRR compared to DVAN's 66.67)

\begin{tabular}{|c|c|c|c|c|c|c|c|}
\hline & Model & $\mathrm{NDCG} \uparrow$ & $\operatorname{MRR} \uparrow$ & $\mathrm{R} @ 1 \uparrow$ & $\mathrm{R} @ 5 \uparrow$ & $\mathrm{R} @ 10 \uparrow$ & Mean $\downarrow$ \\
\hline \multirow{4}{*}{ (a) } & From scratch & 56.20 & 62.25 & 48.16 & 79.57 & 89.01 & 4.31 \\
\hline & Init from VLP & 61.79 & 66.67 & 53.23 & 83.60 & 91.97 & 3.66 \\
\hline & Init from BERT & 63.22 & 67.44 & 54.02 & 83.96 & 92.33 & 3.53 \\
\hline & $\hookrightarrow$ only NSP & 55.89 & 63.15 & 48.98 & 80.45 & 89.72 & 4.15 \\
\hline \multirow{4}{*}{ (b) } & No history & 64.70 & 62.93 & 48.70 & 80.42 & 89.73 & 4.30 \\
\hline & One previous turn & 63.47 & 65.30 & 51.66 & 82.30 & 90.97 & 3.86 \\
\hline & Full history & 63.22 & 67.44 & 54.02 & 83.96 & 92.33 & 3.53 \\
\hline & $\hookrightarrow$ only text & 54.32 & 62.79 & 48.48 & 80.12 & 89.33 & 4.27 \\
\hline \multirow{4}{*}{ (c) } & $\mathrm{CE}$ & 74.47 & 44.94 & 32.23 & 60.10 & 76.70 & 7.57 \\
\hline & ListNet & 74.54 & 46.72 & 33.15 & 61.58 & 77.15 & 7.18 \\
\hline & ListMLE & 72.96 & 36.81 & 20.70 & 54.60 & 73.28 & 8.90 \\
\hline & ApproxNDCG & 72.45 & 49.88 & 37.88 & 62.90 & $\mathbf{7 7 . 4 0}$ & 7.26 \\
\hline \multirow{4}{*}{ (d) } & ЕРОСН & 74.84 & 47.40 & 34.30 & 61.58 & 77.78 & 7.12 \\
\hline & LENGTH & 75.07 & 47.33 & 33.88 & 62.20 & 78.50 & 7.01 \\
\hline & RANK & 75.13 & 50.00 & 38.28 & 60.93 & 77.28 & 6.90 \\
\hline & DIVERSE & 75.35 & 51.17 & 38.90 & 62.82 & 77.98 & 6.69 \\
\hline
\end{tabular}

Table 3: Extensive ablation studies: training with (a) various settings and (b) contexts on v1.0 val; dense annotation fine-tuning with (c) varying ranking methods and (d) various ensemble strategies on v1.0 test-std.

and comparable results with the state of the art in the generative setting (e.g., 55.95 MRR score vs. DVAN's 55.94). This validates the effectiveness of our VD-BERT in both settings using a unified Transformer encoder. By contrast, VisDial-BERT can only support the discriminative setting.

\subsection{Ablation Study}

We first study how different training settings influence the results in Table 3(a). We observe that initializing the model with weights from BERT indeed benefits the visual dialog task a lot, increasing the NDCG score by about $7 \%$ absolute over the model trained from scratch. Surprisingly, the model initialized with the weights from VLP that was pretrained on Conceptual Captions (Sharma et al., 2018), does not work better than the one initialized from BERT. It might be due to the domain discrepancy between image captions and multi-turn dialogs, as well as the slightly different experiment settings (e.g., we extract 36 objects from image compared to their 100 objects). Another possible reason might be that the VisDial data with more than one million image-dialog turn pairs can provide adequate contexts to adapt BERT for effective vision and dialog fusion. We also find that the visually grounded MLM is crucial for transferring BERT into the multimodal setting, indicated by a large performance drop when using only NSP.

We then examine the impact of varying the dialog context used for training in Table 3(b). With longer dialog history ("Full history"), our model indeed yields better results in most of the ranking metrics, while the one without using any dialog history obtains the highest NDCG score. This in- 

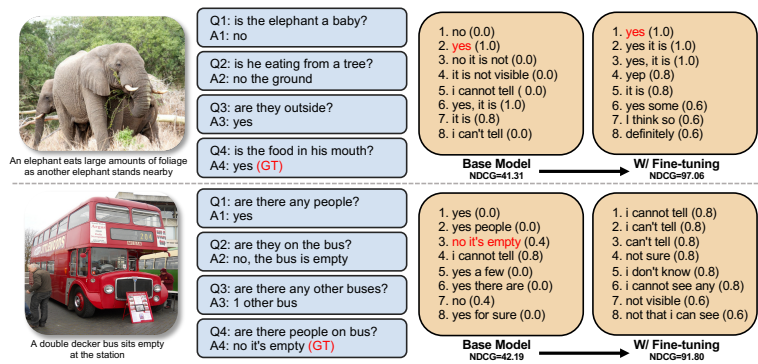

Figure 3: The effects of dense annotation fine-tuning in our VD-BERT for two examples. GT: ground truth.

dicates that dense relevance scores might be annotated with less consideration of dialog history. If we remove the visual cues from the "Full history" model, we see a drop in all metrics, especially, on NDCG. However, this version still obtains comparable results to the "No history" variant, revealing that textual information dominates the VisDial task

In Table 3(c), we compare Cross Entropy (CE) training with a bunch of other listwise ranking optimization methods: ListNet (Cao et al., 2007), ListMLE (Xia et al., 2008), and approxNDCG (Qin et al., 2010). Among these methods, ListNet yields the best NDCG and Mean Rank, while the approxNDCG achieves the best MRR and Recall on VisDial v1.0 test-std. Therefore, we employ the ListNet as our ranking module.

We also explore ways to achieve the best ensemble performance with various model selection criteria in Table 3(d). We consider three criteria, EPOCH, LENGTH, and RANK that respectively refer to predictions from different epochs of a single model, from different models trained with varying context lengths and with different ranking methods in Table 3(b)-(c). We use four predictions from each criterion and combine their diverse predictions (DIVERSE) by summing up their normalized ranking scores. We observe that EPOCH contributes the least to the ensemble performance while RANK models are more helpful than LENGTH models. The diverse set of them leads to the best performance.

\subsection{Fine-tuning on Dense Annotations}

In this section, we focus on the effect of dense annotation fine-tuning and try to analyze the reason of the inconsistency issue between NDCG and other ranking metrics (see Table 1) in the following.

Case Study. We provide two examples to qualitatively demonstrate how dense annotation finetuning results in better NDCG scores in Figure 3. For the example at the top, fine-tuning helps our model to assign higher ranks to the answers that share similar semantics with the ground truth answer and should also be regarded as correct ("yes, it is" and "yep" vs. "yes"). In the example at the bottom, we spot a mismatch between the sparse and dense annotations: the ground truth answer "no, it's empty" is only given a 0.4 relevance score, while uncertain answers like "i don't know" are considered to be more relevant. In this case, fine-tuning instead makes our model fail to predict the correct answer despite the increase of NDCG score.

Relevance Score and Question Type Analysis. We first show how various metrics change for finetuning in Figure 4. For this experiment, we randomly sample 200 instances from VisDial v1.0 val as the test data and use the rest for fine-tuning with the ListNet ranking method. We observe that NDCG keeps increasing with more epochs of fine-tuning, while other metrics such as Recall@K and MRR) drop. For further analysis, we classify the 2,064 instances in VisDial v1.0 val set based on the ground-truth's relevance score and question type (Table 4). We consider four bins $\{0.0,0.2 \sim 0.4,0.6 \sim 0.8,1.0\}$ for the relevance score and four question types: Yes/no, Number, Color, and Others. We then analyze the NDCG scores assigned by DAN (Kang et al., 2019) and our VD-BERT with and without dense annotation fine-tuning. We choose DAN as it achieves good NDCG scores (Table 1) and provides the source code to reproduce their predictions.

By examining the distribution of the relevance scores, we find that only $31 \%$ of them are aligned well with the sparse annotations and $9 \%$ are totally misaligned. As the degree of such mismatch increases (relevance score changes $1.0 \rightarrow 0.0$ ), both DAN and our model witness a plunge in NDCG $(63.29 \rightarrow 43.86$ and $70.25 \rightarrow 48.07)$, while dense annotation fine-tuning significantly boosts NDCG scores for all groups, especially for the most misaligned one $(48.07 \rightarrow 82.84$ for our model). These results validate that the misalignment of the sparse and dense annotations is the key reason for the inconsistency between NDCG and other metrics.

For question types, we observe that Yes/no is the major type $(76 \%)$ and also the easiest one, while Number is the most challenging and least frequent one $(3 \%)$. Our model outperforms DAN by over $10 \%$ in most of the question types except Color. Fine-tuning on dense annotations gives our model huge improvements across all the question types, especially for Others with over $30 \%$ absolute gain. 


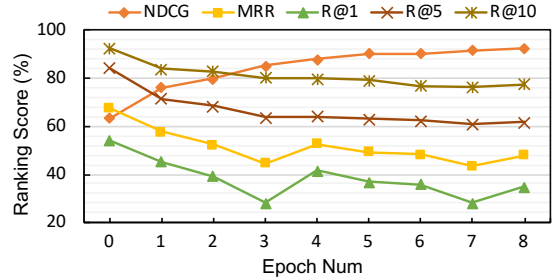

Figure 4: Dense annotation fine-tuning on various metrics with the ListNet method.

\begin{tabular}{|c|c|c|c|c|c|c|c|c|c|}
\hline \multirow[b]{2}{*}{ Models } & \multirow[b]{2}{*}{ All } & \multicolumn{4}{|c|}{ Relevance Score } & \multicolumn{4}{|c|}{ Question Type } \\
\hline & & $\begin{array}{c}1.0 \\
(31 \%)\end{array}$ & $\begin{array}{c}0.6 \sim 0.8 \\
(35 \%)\end{array}$ & $\begin{array}{c}0.2 \sim 0.4 \\
(25 \%)\end{array}$ & $\begin{array}{c}0.0 \\
(9 \%)\end{array}$ & $\begin{array}{l}\text { Yes/no } \\
(76 \%)\end{array}$ & $\begin{array}{c}\text { Number } \\
(3 \%)\end{array}$ & $\begin{array}{l}\text { Color } \\
(11 \%)\end{array}$ & $\begin{array}{l}\text { Others } \\
(10 \%)\end{array}$ \\
\hline DAN & 58.28 & 63.29 & 61.02 & 53.29 & 43.86 & 59.86 & 41.03 & 57.55 & 51.89 \\
\hline Ours & 63.55 & 70.25 & 65.18 & 58.40 & 48.07 & 65.45 & 48.98 & 58.51 & 58.75 \\
\hline Ours (w/ft) & 89.62 & 95.38 & 89.76 & 84.63 & 82.84 & 91.05 & 74.41 & 84.00 & 89.12 \\
\hline
\end{tabular}

Table 4: NDCG scores in VisDial v1.0 val split broken down into 4 groups based on relevance score and the question type. The $\%$ value in the parentheses denotes the corresponding data proportion.
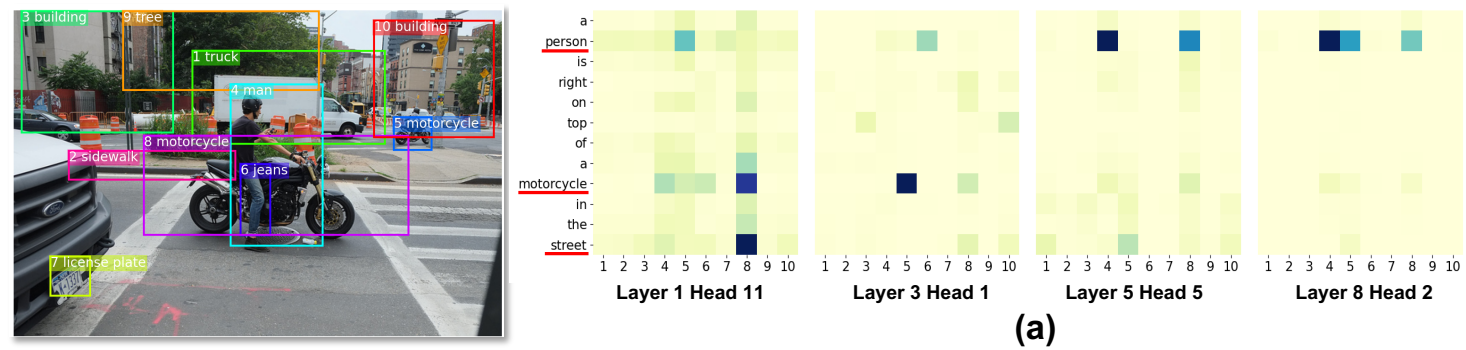

(a)

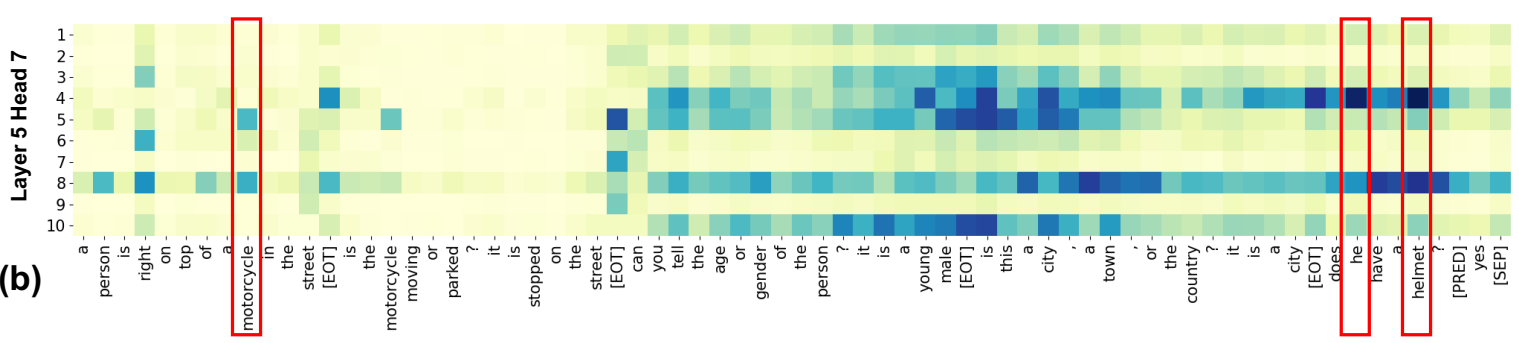

Figure 5: Attention weight visualization in our VD-BERT for a sampled image-dialog example.

\subsection{Attention Visualization}

To interpret our VD-BERT, we visualize the attention weights on the top 10 detected objects from its caption in Figure 5(a). We observe that many heads at different layers can correctly ground some entities like person and motorcycle in the image, and even reveal some high-level semantic correlations such as person $\leftrightarrow$ motorcycle (at L8H2) and motorcycle $\leftrightarrow$ street (at L1H11). Besides, heads at higher layers tend to have a sharper focus on specific objects like the man and the motorcycles in the image.

Next, we examine how our VD-BERT captures the interactions between image and multi-turn dialog. In contrast to other vision-language tasks, visual dialog has a more complex multi-turn structure, thereby posing a hurdle for effective fusion. As shown in Figure 5(b), VD-BERT can ground entities and discover some object relations, e.g., helmet is precisely related to the man and the motorcycle in the image (see the rightmost red box). More interestingly, it can even resolve visual pronoun coreference of he in the question to the man in the image (see the middle red box). We provide more qualitative examples in Figure 6 and 7.

\section{Conclusion}

We have presented VD-BERT, a unified visiondialog Transformer model that exploits the pretrained BERT language models for visual dialog. VD-BERT is capable of modeling all the interactions between an image and a multi-turn dialog within a single-stream Transformer encoder and enables the effective fusion of features from both modalities via simple visually grounded training. Besides, it can either rank or generate answers seamlessly. Without pretraining on external visionlanguage datasets, our model establishes new stateof-the-art performance in the discriminative setting and shows promising results in the generative setting on the visual dialog benchmarks.

\section{Acknowledgements}

We thank Chien-Sheng Wu, Jiashi Feng, Jiaxin Qi, and our anonymous reviewers for their insightful feedback on our paper. This work was partially supported by the Research Grants Council of the Hong Kong Special Administrative Region, China (No. CUHK 14210717, General Research Fund; No. CUHK 2300174, Collaborative Research Fund). 


\section{References}

Shubham Agarwal, Trung Bui, Joon-Young Lee, Ioannis Konstas, and Verena Rieser. 2020. History for visual dialog: Do we really need it? In Proceedings of the 58th Annual Meeting of the Association for Computational Linguistics, ACL 2020, Online, July 5-10, 2020, pages 8182-8197. Association for Computational Linguistics.

Chris Alberti, Jeffrey Ling, Michael Collins, and David Reitter. 2019. Fusion of detected objects in text for visual question answering. In Proceedings of the 2019 Conference on Empirical Methods in Natural Language Processing and the 9th International Joint Conference on Natural Language Processing, EMNLP-IJCNLP 2019, Hong Kong, China, November 3-7, 2019, pages 2131-2140. Association for Computational Linguistics.

Stanislaw Antol, Aishwarya Agrawal, Jiasen Lu, Margaret Mitchell, Dhruv Batra, C. Lawrence Zitnick, and Devi Parikh. 2015. VQA: visual question answering. In 2015 IEEE International Conference on Computer Vision, ICCV 2015, Santiago, Chile, December 7-13, 2015, pages 2425-2433.

Lei Jimmy Ba, Jamie Ryan Kiros, and Geoffrey E. Hinton. 2016. Layer normalization. CoRR, abs/1607.06450.

Dzmitry Bahdanau, Kyunghyun Cho, and Yoshua Bengio. 2015. Neural machine translation by jointly learning to align and translate. In 3rd International Conference on Learning Representations, ICLR 2015, San Diego, CA, USA, May 7-9, 2015, Conference Track Proceedings.

Zhe Cao, Tao Qin, Tie-Yan Liu, Ming-Feng Tsai, and Hang Li. 2007. Learning to rank: from pairwise approach to listwise approach. In Machine Learning, Proceedings of the Twenty-Fourth International Conference (ICML 2007), Corvallis, Oregon, USA, June 20-24, 2007, volume 227 of ACM International Conference Proceeding Series, pages 129-136. ACM.

Yen-Chun Chen, Linjie Li, Licheng Yu, Ahmed El Kholy, Faisal Ahmed, Zhe Gan, Yu Cheng, and Jingjing Liu. 2019. UNITER: learning universal image-text representations. CoRR, abs/1909.11740.

Abhishek Das, Satwik Kottur, Khushi Gupta, Avi Singh, Deshraj Yadav, José M. F. Moura, Devi Parikh, and Dhruv Batra. 2017. Visual dialog. In 2017 IEEE Conference on Computer Vision and Pattern Recognition, CVPR 2017, Honolulu, HI, USA, July 21-26, 2017, pages 1080-1089.

Jacob Devlin, Ming-Wei Chang, Kenton Lee, and Kristina Toutanova. 2019. BERT: pre-training of deep bidirectional transformers for language understanding. In Proceedings of the 2019 Conference of the North American Chapter of the Association for Computational Linguistics: Human Language Technologies, NAACL-HLT 2019, Minneapolis, MN,
USA, June 2-7, 2019, Volume 1 (Long and Short Papers), pages 4171-4186.

Li Dong, Nan Yang, Wenhui Wang, Furu Wei, Xiaodong Liu, Yu Wang, Jianfeng Gao, Ming Zhou, and Hsiao-Wuen Hon. 2019. Unified language model pre-training for natural language understanding and generation. In Advances in Neural Information Processing Systems 32: Annual Conference on Neural Information Processing Systems 2019, NeurIPS 2019, 8-14 December 2019, Vancouver, BC, Canada, pages 13042-13054.

Zhe Gan, Yu Cheng, Ahmed El Kholy, Linjie Li, Jingjing Liu, and Jianfeng Gao. 2019. Multi-step reasoning via recurrent dual attention for visual dia$\log$. In Proceedings of the 57th Conference of the Association for Computational Linguistics, ACL 2019, Florence, Italy, July 28-August 2, 2019, Volume 1: Long Papers, pages 6463-6474.

Dalu Guo, Chang Xu, and Dacheng Tao. 2019a. Imagequestion-answer synergistic network for visual dialog. In IEEE Conference on Computer Vision and Pattern Recognition, CVPR 2019, Long Beach, CA, USA, June 16-20, 2019, pages 10434-10443.

Dan Guo, Hui Wang, and Meng Wang. 2019b. Dual visual attention network for visual dialog. In Proceedings of the Twenty-Eighth International Joint Conference on Artificial Intelligence, IJCAI 2019, Macao, China, August 10-16, 2019, pages 4989-4995. ijcai.org.

Dan Guo, Hui Wang, Hanwang Zhang, Zheng-Jun Zha, and Meng Wang. 2020. Iterative contextaware graph inference for visual dialog. In 2020 IEEE/CVF Conference on Computer Vision and Pattern Recognition, CVPR 2020, Seattle, WA, USA, June 13-19, 2020, pages 10052-10061. IEEE.

Ronghang $\mathrm{Hu}$, Jacob Andreas, Marcus Rohrbach, Trevor Darrell, and Kate Saenko. 2017. Learning to reason: End-to-end module networks for visual question answering. In IEEE International Conference on Computer Vision, ICCV 2017, Venice, Italy, October 22-29, 2017, pages 804-813. IEEE Computer Society.

Xiaoze Jiang, Jing Yu, Zengchang Qin, Yingying Zhuang, Xingxing Zhang, Yue Hu, and Qi Wu. 2020. Dualvd: An adaptive dual encoding model for deep visual understanding in visual dialogue. In The Thirty-Fourth AAAI Conference on Artificial Intelligence, AAAI 2020, The Thirty-Second Innovative Applications of Artificial Intelligence Conference, IAAI 2020, The Tenth AAAI Symposium on Educational Advances in Artificial Intelligence, EAAI 2020, New York, NY, USA, February 7-12, 2020, pages 1112511132. AAAI Press.

Gi-Cheon Kang, Jaeseo Lim, and Byoung-Tak Zhang. 2019. Dual attention networks for visual reference resolution in visual dialog. In Proceedings of the 2019 Conference on Empirical Methods in Natural Language Processing and the 9th International 
Joint Conference on Natural Language Processing, EMNLP-IJCNLP 2019, Hong Kong, China, November 3-7, 2019, pages 2024-2033. Association for Computational Linguistics.

Gi-Cheon Kang, Junseok Park, Hwaran Lee, ByoungTak Zhang, and Jin-Hwa Kim. 2020. Dialgraph: Sparse graph learning networks for visual dialog. CoRR, abs/2004.06698.

Hyounghun Kim, Hao Tan, and Mohit Bansal. 2020. Modality-balanced models for visual dialogue. In The Thirty-Fourth AAAI Conference on Artificial Intelligence, AAAI 2020, The Thirty-Second Innovative Applications of Artificial Intelligence Conference, IAAI 2020, The Tenth AAAI Symposium on Educational Advances in Artificial Intelligence, EAAI 2020, New York, NY, USA, February 7-12, 2020 , pages 8091-8098. AAAI Press.

Diederik P. Kingma and Jimmy Ba. 2015. Adam: A method for stochastic optimization. In 3rd International Conference on Learning Representations, ICLR 2015, San Diego, CA, USA, May 7-9, 2015, Conference Track Proceedings.

Satwik Kottur, José M. F. Moura, Devi Parikh, Dhruv Batra, and Marcus Rohrbach. 2018. Visual coreference resolution in visual dialog using neural module networks. In Computer Vision - ECCV 2018 - 15th European Conference, Munich, Germany, September 8-14, 2018, Proceedings, Part XV, pages 160178

Ranjay Krishna, Yuke Zhu, Oliver Groth, Justin Johnson, Kenji Hata, Joshua Kravitz, Stephanie Chen, Yannis Kalantidis, Li-Jia Li, David A. Shamma, Michael S. Bernstein, and Li Fei-Fei. 2017. Visual genome: Connecting language and vision using crowdsourced dense image annotations. International Journal of Computer Vision, 123(1):32-73.

Gen Li, Nan Duan, Yuejian Fang, Ming Gong, and Daxin Jiang. 2020. Unicoder-vl: A universal encoder for vision and language by cross-modal pretraining. In The Thirty-Fourth AAAI Conference on Artificial Intelligence, AAAI 2020, The ThirtySecond Innovative Applications of Artificial Intelligence Conference, IAAI 2020, The Tenth AAAI Symposium on Educational Advances in Artificial Intelligence, EAAI 2020, New York, NY, USA, February 7-12, 2020, pages 11336-11344. AAAI Press.

Liunian Harold Li, Mark Yatskar, Da Yin, Cho-Jui Hsieh, and Kai-Wei Chang. 2019. Visualbert: A simple and performant baseline for vision and language. CoRR, abs/1908.03557.

Jiasen Lu, Dhruv Batra, Devi Parikh, and Stefan Lee. 2019. Vilbert: Pretraining task-agnostic visiolinguistic representations for vision-and-language tasks. In Advances in Neural Information Process ing Systems 32: Annual Conference on Neural Information Processing Systems 2019, NeurIPS 2019, 8 14 December 2019, Vancouver, BC, Canada, pages 13-23.
Jiasen Lu, Anitha Kannan, Jianwei Yang, Devi Parikh, and Dhruv Batra. 2017. Best of both worlds: Transferring knowledge from discriminative learning to a generative visual dialog model. In Advances in Neural Information Processing Systems 30: Annual Conference on Neural Information Processing Systems 2017, 4-9 December 2017, Long Beach, CA, USA, pages 314-324.

Vishvak Murahari, Dhruv Batra, Devi Parikh, and Abhishek Das. 2019. Large-scale pretraining for visual dialog: A simple state-of-the-art baseline. CoRR, abs/1912.02379.

Van-Quang Nguyen, Masanori Suganuma, and Takayuki Okatani. 2019. Efficient attention mechanism for handling all the interactions betweenn many inputs with application to visual dialog. CoRR, abs/1911.11390.

Yulei Niu, Hanwang Zhang, Manli Zhang, Jianhong Zhang, Zhiwu Lu, and Ji-Rong Wen. 2019. Recursive visual attention in visual dialog. In IEEE Conference on Computer Vision and Pattern Recognition, CVPR 2019, Long Beach, CA, USA, June 16-20, 2019, pages 6679-6688.

Sungjin Park, Taesun Whang, Yeochan Yoon, and Hueiseok Lim. 2020. Multi-view attention networks for visual dialog. CoRR, abs/2004.14025.

Jiaxin Qi, Yulei Niu, Jianqiang Huang, and Hanwang Zhang. 2020. Two causal principles for improving visual dialog. In 2020 IEEE/CVF Conference on Computer Vision and Pattern Recognition, CVPR 2020, Seattle, WA, USA, June 13-19, 2020, pages 10857-10866. IEEE.

Tao Qin, Tie-Yan Liu, and Hang Li. 2010. A general approximation framework for direct optimization of information retrieval measures. Inf. Retr., 13(4):375-397.

Shaoqing Ren, Kaiming He, Ross B. Girshick, and Jian Sun. 2015. Faster R-CNN: towards real-time object detection with region proposal networks. In Advances in Neural Information Processing Systems 28: Annual Conference on Neural Information Processing Systems 2015, December 7-12, 2015, Montreal, Quebec, Canada, pages 91-99.

Idan Schwartz, Seunghak Yu, Tamir Hazan, and Alexander G. Schwing. 2019. Factor graph attention. In IEEE Conference on Computer Vision and Pattern Recognition, CVPR 2019, Long Beach, CA, USA, June 16-20, 2019, pages 2039-2048.

Paul Hongsuck Seo, Andreas M. Lehrmann, Bohyung Han, and Leonid Sigal. 2017. Visual reference resolution using attention memory for visual dialog. In Advances in Neural Information Processing Systems 30: Annual Conference on Neural Information Processing Systems 2017, 4-9 December 2017, Long Beach, CA, USA, pages 3719-3729. 
Piyush Sharma, Nan Ding, Sebastian Goodman, and Radu Soricut. 2018. Conceptual captions: A cleaned, hypernymed, image alt-text dataset for automatic image captioning. In Proceedings of the 56th Annual Meeting of the Association for Computational Linguistics, ACL 2018, Melbourne, Australia, July 15-20, 2018, Volume 1: Long Papers, pages 2556-2565.

Weijie Su, Xizhou Zhu, Yue Cao, Bin Li, Lewei Lu, Furu Wei, and Jifeng Dai. 2020. VL-BERT: pretraining of generic visual-linguistic representations. In 8th International Conference on Learning Representations, ICLR 2020, Addis Ababa, Ethiopia, April 26-30, 2020. OpenReview.net.

Alane Suhr, Stephanie Zhou, Ally Zhang, Iris Zhang, Huajun Bai, and Yoav Artzi. 2019. A corpus for reasoning about natural language grounded in photographs. In Proceedings of the 57th Conference of the Association for Computational Linguistics, ACL 2019, Florence, Italy, July 28-August 2, 2019, Volume 1: Long Papers, pages 6418-6428.

Chen Sun, Austin Myers, Carl Vondrick, Kevin Murphy, and Cordelia Schmid. 2019. Videobert: A joint model for video and language representation learning. In 2019 IEEE/CVF International Conference on Computer Vision, ICCV 2019, Seoul, Korea (South), October 27 - November 2, 2019, pages 7463-7472. IEEE.

Ilya Sutskever, Oriol Vinyals, and Quoc V. Le. 2014. Sequence to sequence learning with neural networks. In Advances in Neural Information Processing Systems 27: Annual Conference on Neural Information Processing Systems 2014, December 8-13 2014, Montreal, Quebec, Canada, pages 3104-3112.

Hao Tan and Mohit Bansal. 2019. LXMERT: learning cross-modality encoder representations from transformers. In Proceedings of the 2019 Conference on Empirical Methods in Natural Language Processing and the 9th International Joint Conference on Natural Language Processing, EMNLP-IJCNLP 2019, Hong Kong, China, November 3-7, 2019, pages 5099-5110. Association for Computational Linguistics.

Ashish Vaswani, Noam Shazeer, Niki Parmar, Jakob Uszkoreit, Llion Jones, Aidan N. Gomez, Lukasz Kaiser, and Illia Polosukhin. 2017. Attention is all you need. In Advances in Neural Information Processing Systems 30: Annual Conference on Neural Information Processing Systems 2017, 4-9 December 2017, Long Beach, CA, USA, pages 5998-6008.

Taesun Whang, Dongyub Lee, Chanhee Lee, Kisu Yang, Dongsuk Oh, and Heuiseok Lim. 2019. Domain adaptive training BERT for response selection. CoRR, abs/1908.04812.

Qi Wu, Peng Wang, Chunhua Shen, Ian D. Reid, and Anton van den Hengel. 2018. Are you talking to me? reasoned visual dialog generation through adversarial learning. In 2018 IEEE Conference on Computer
Vision and Pattern Recognition, CVPR 2018, Salt Lake City, UT, USA, June 18-22, 2018, pages 61066115.

Yonghui Wu, Mike Schuster, Zhifeng Chen, Quoc V. Le, Mohammad Norouzi, Wolfgang Macherey, Maxim Krikun, Yuan Cao, Qin Gao, Klaus Macherey, Jeff Klingner, Apurva Shah, Melvin Johnson, Xiaobing Liu, Lukasz Kaiser, Stephan Gouws, Yoshikiyo Kato, Taku Kudo, Hideto Kazawa, Keith Stevens, George Kurian, Nishant Patil, Wei Wang, Cliff Young, Jason Smith, Jason Riesa, Alex Rudnick, Oriol Vinyals, Greg Corrado, Macduff Hughes, and Jeffrey Dean. 2016. Google's neural machine translation system: Bridging the gap between human and machine translation. CoRR, abs/1609.08144.

Fen Xia, Tie-Yan Liu, Jue Wang, Wensheng Zhang, and Hang Li. 2008. Listwise approach to learning to rank: theory and algorithm. In Machine Learning, Proceedings of the Twenty-Fifth International Conference (ICML 2008), Helsinki, Finland, June 5-9, 2008, volume 307 of ACM International Conference Proceeding Series, pages 1192-1199. ACM.

Tianhao Yang, Zheng-Jun Zha, and Hanwang Zhang. 2019. Making history matter: History-advantage sequence training for visual dialog. In 2019 IEEE/CVF International Conference on Computer Vision, ICCV 2019, Seoul, Korea (South), October 27 - November 2, 2019, pages 2561-2569. IEEE.

Peter Young, Alice Lai, Micah Hodosh, and Julia Hockenmaier. 2014. From image descriptions to visual denotations: New similarity metrics for semantic inference over event descriptions. TACL, 2:67-78.

Rowan Zellers, Yonatan Bisk, Ali Farhadi, and Yejin Choi. 2019. From recognition to cognition: Visual commonsense reasoning. In IEEE Conference on Computer Vision and Pattern Recognition, CVPR 2019, Long Beach, CA, USA, June 16-20, 2019, pages 6720-6731. Computer Vision Foundation / IEEE.

Zilong Zheng, Wenguan Wang, Siyuan Qi, and SongChun Zhu. 2019. Reasoning visual dialogs with structural and partial observations. In IEEE Conference on Computer Vision and Pattern Recognition, CVPR 2019, Long Beach, CA, USA, June 16-20, 2019, pages 6669-6678.

Luowei Zhou, Hamid Palangi, Lei Zhang, Houdong $\mathrm{Hu}$, Jason J. Corso, and Jianfeng Gao. 2020. Unified vision-language pre-training for image captioning and VQA. In The Thirty-Fourth AAAI Conference on Artificial Intelligence, AAAI 2020, The Thirty-Second Innovative Applications of Artificial Intelligence Conference, IAAI 2020, The Tenth AAAI Symposium on Educational Advances in Artificial Intelligence, EAAI 2020, New York, NY, USA, February 7-12, 2020, pages 13041-13049. AAAI Press. 
(a)

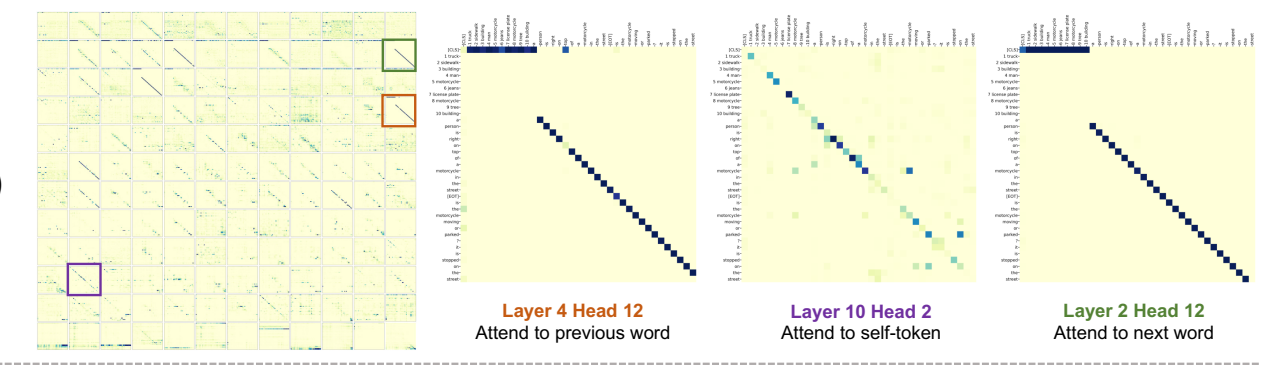

(b)
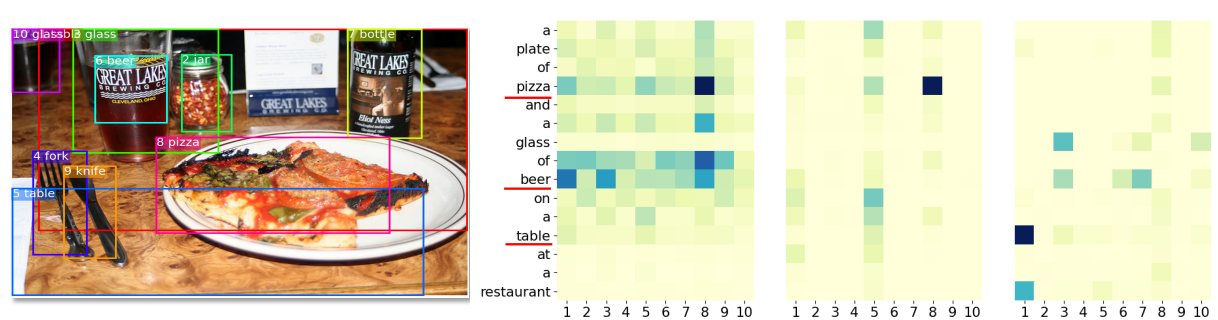

L1H4

L2H4

L5H1

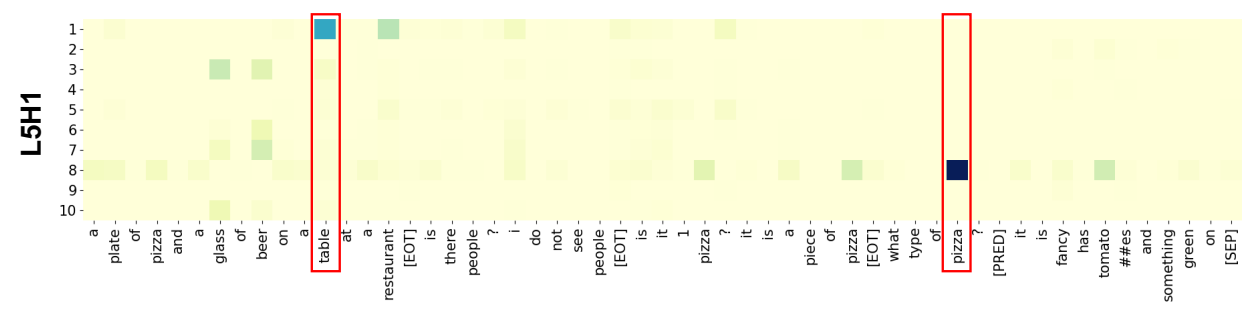

(c)
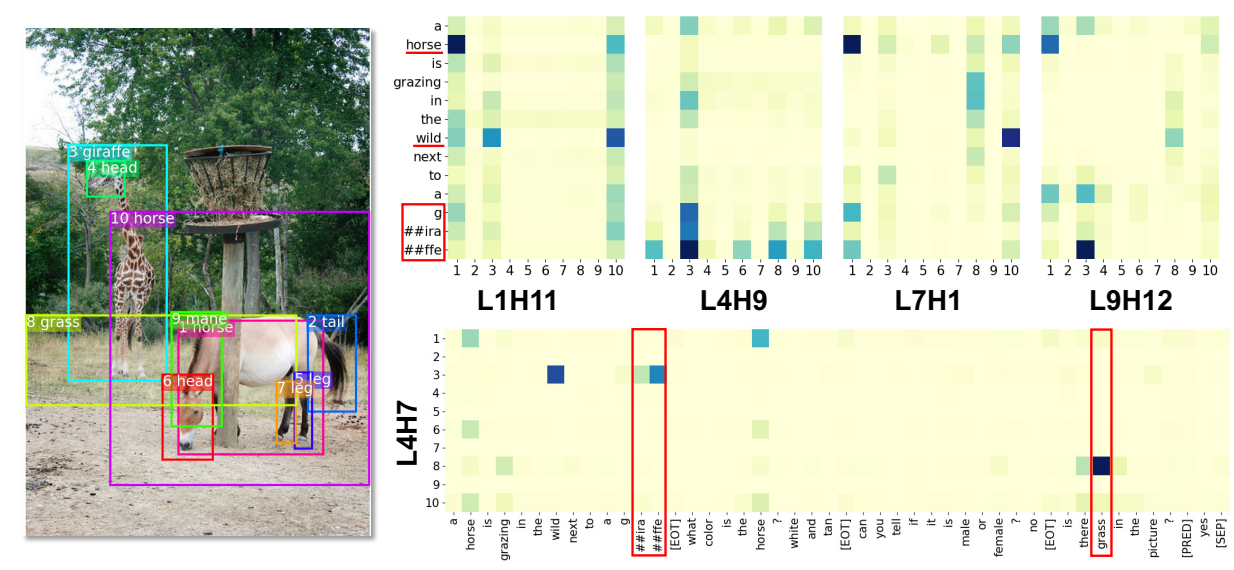

(d)
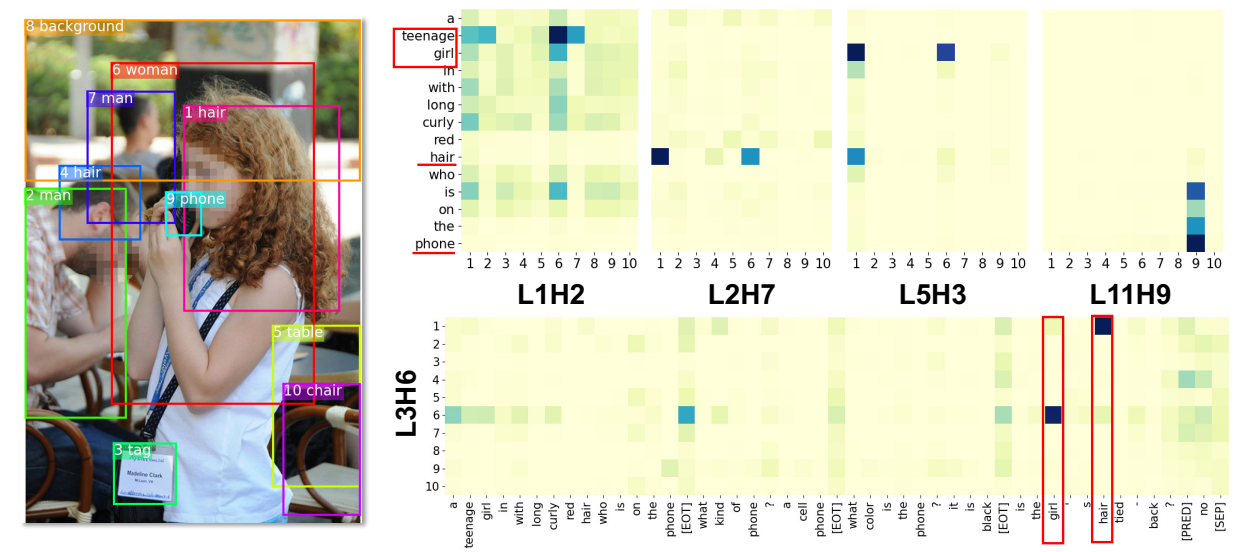

Figure 6: More attention visualization examples showing that VD-BERT achieves the effective fusion of vision and dialog contents. L $x \mathrm{H} y$ : Layer $x$ Head $y(1 \leq x, y \leq 12)$. (a) It learns three apparent attention patterns for the example in Figure 5: attentions that a token puts to its previous token, to itself, and to the next token. Besides, some of its attention heads can precisely ground some entities between image and caption/multi-turn dialog: (b) pizza, beer, and table; (c) horse, wild, and giraffe; (d) teenage girl, hair, and phone. 


\begin{tabular}{|c|c|c|c|c|}
\hline Image + Caption & GT Dialog & DAN & VD-BERT & VD-BERT (w/ft) \\
\hline $\begin{array}{l}\text { a bro } \\
\text { corner }\end{array}$ & 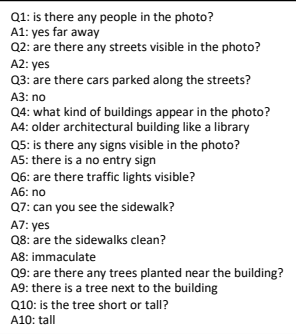 & 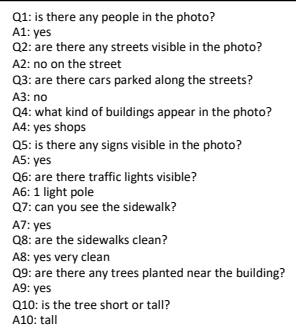 & $\begin{array}{l}\text { Q1: is there any people in the photo? } \\
\text { A: ves } \\
\text { Q2: are there any streets visible in the photo? } \\
\text { A2: ves } \\
\text { Q3: are there cars parked along the streets? } \\
\text { A3: no } \\
\text { Q4 what kind of buildings appear in the photo? } \\
\text { A: older architectural building like a library } \\
\text { Q5: is there any signs visible in the photo? } \\
\text { A: ves } \\
\text { Q6: are there traffic lights visible? } \\
\text { A6: no } \\
\text { Q7 can you see the sidewalk? } \\
\text { A: ves } \\
\text { Q8: are the sidewalks clean? } \\
\text { A: ves } \\
\text { Q9: are there any trees planted near the building? } \\
\text { A: there is a tree next to the building } \\
\text { Q10: is the tree short or tall? } \\
\text { A10: tall }\end{array}$ & 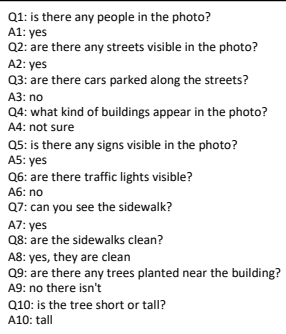 \\
\hline & 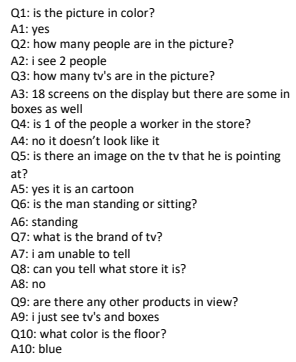 & 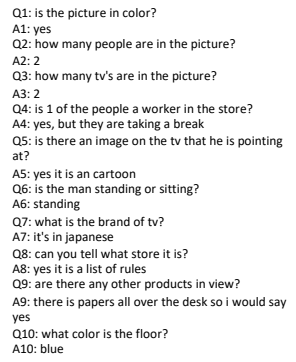 & 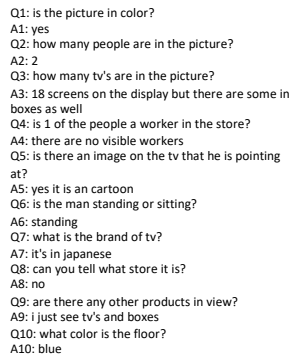 & 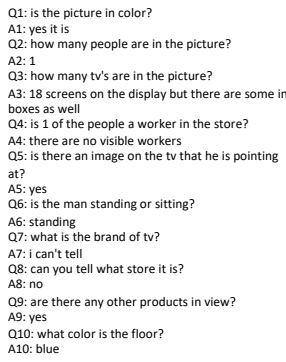 \\
\hline & 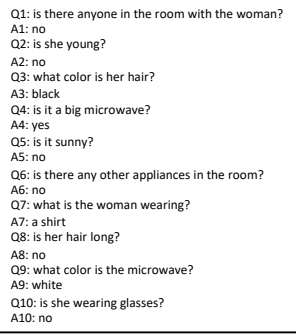 & 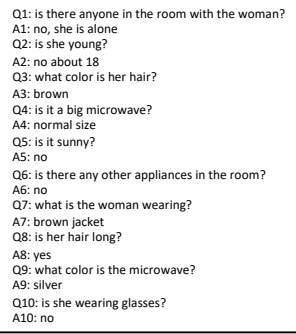 & 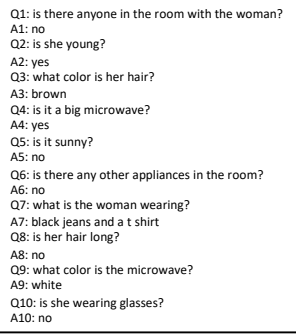 & 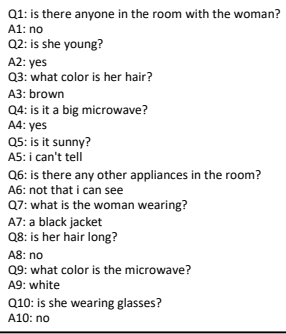 \\
\hline an el & 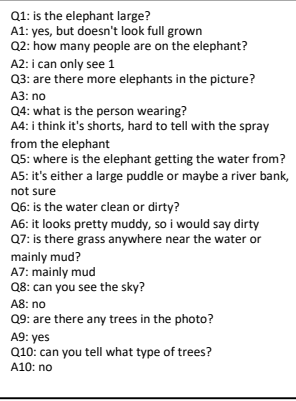 & 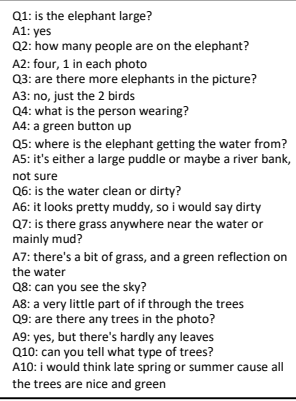 & 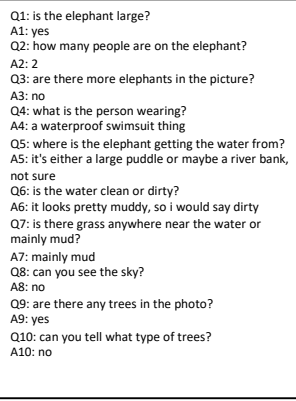 & 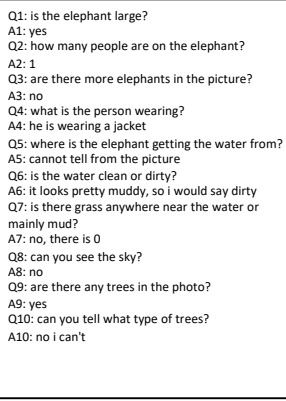 \\
\hline $\begin{array}{l}\text { a man wearing a hat hold } \\
\text { white dog }\end{array}$ & 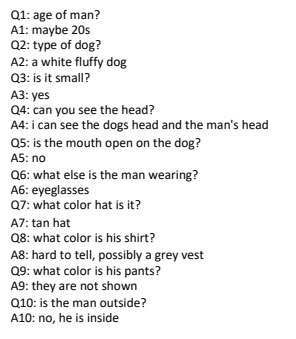 & 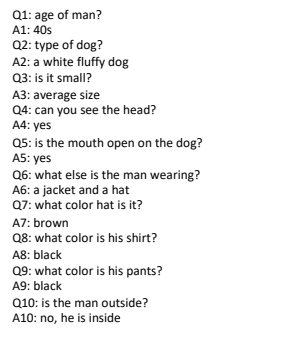 & 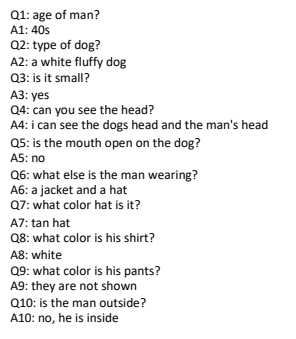 & 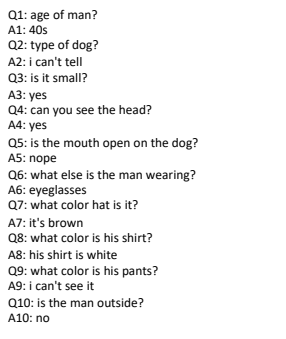 \\
\hline laun & 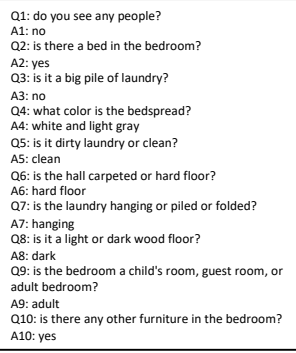 & 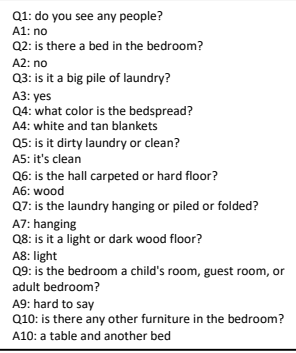 & 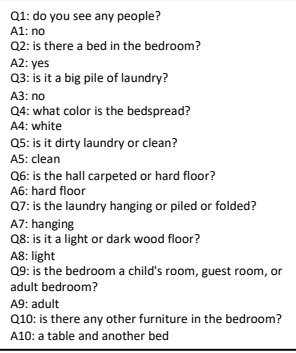 & 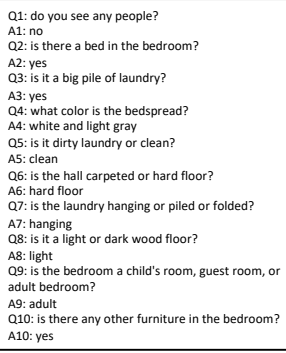 \\
\hline
\end{tabular}

Figure 7: More qualitative examples in VisDial v1.0 val split for three model variants: DAN (Kang et al., 2019), VD-BERT, and VD-BERT with dense annotation fine-tuning. The second column is for ground truth (GT) dialog. 